\title{
Identifying association model for single-nucleotide polymorphisms of ORAl1 gene for breast cancer
}

\author{
Wei-Chiao Chang ${ }^{1,2,3,9 \dagger}$, Yong-Yuan Fang ${ }^{4 \dagger}$, Hsueh-Wei Chang ${ }^{3,5,6^{*}}$, Li-Yeh Chuang ${ }^{7}$, Yu-Da Lin ${ }^{8}$, \\ Ming-Feng $\mathrm{Hou}^{3,9,10^{*}}$ and Cheng-Hong Yang ${ }^{8^{*}}$
}

\begin{abstract}
Background: ORA/1 channels play an important role for breast cancer progression and metastasis. Previous studies indicated the strong correlation between breast cancer and individual single nucleotide polymorphisms (SNPs) of ORAll gene. However, the possible SNP-SNP interaction of ORAll gene was not investigated.

Results: To develop the complex analyses of SNP-SNP interaction, we propose a genetic algorithm (GA) to detect the model of breast cancer association between five SNPs (rs12320939, rs12313273, rs7135617, rs6486795 and rs712853) of ORAl1 gene. For individual SNPS, the differences between case and control groups in five SNPs of ORAl1 gene were not significant. In contrast, GA-generated SNP models show that 2-SNP (rs12320939-GT/rs6486795-CT), 3-SNP (rs12320939GT/rs12313273-TT/rs6486795-TC), 5-SNP (rs12320939-GG/rs12313273-TC/rs7135617-TT/rs6486795-TT/rs712853-TT) have higher risks for breast cancer in terms of odds ratio analysis (1.357, 1.689, and 13.148, respectively).

Conclusion: Taken together, the cumulative effects of SNPS of ORA/1 gene in breast cancer association study were well demonstrated in terms of GA-generated SNP models.
\end{abstract}

Keywords: Single nucleotide polymorphism, Genetic algorithm, SNP interaction, Breast cancer

\section{Background}

Single nucleotide polymorphisms (SNPs) are one of the most common variants in human genome [1]. Currently, SNPs have been applied to the association studies for complex diseases [2-4]. Genome-wide association studies (GWAS) can identify the several SNPs predisposing to many diseases [5-8]. Although GWAS covers human genome-wide SNPs, many SNPs of non-significance are commonly ignored. Recently, the possible jointed effects of gene-gene interactions are gradually uncovered in predicting many disease risks [9-12]. However, when simultaneously evaluate the complex interactions amongst huge SNPs, these interactions are complex and it may need the help of new strategy [13] or computation [14].

Similarly, the non-GWAS association studies have the similar condition to ignore the possible gene-gene

\footnotetext{
* Correspondence: changhw@kmu.edu.tw; mifeho@kmu.edu.tw; chyang@cc.kuas.edu.tw

${ }^{\dagger}$ Equal contributors

${ }^{3}$ Cancer Center, Kaohsiung Medical University Hospital, Kaohsiung, Taiwan ${ }^{8}$ Department of Electronic Engineering, National Kaohsiung University of Applied Sciences, Kaohsiung, Taiwan

Full list of author information is available at the end of the article
}

interactions. For example, several individual SNPs of the ORAI calcium release-activated calcium modulator 1 (ORAI1) gene have reported to be involved in breast cancer susceptibility [15]. However, the possible SNP-SNP interactions of ORAI1 gene associated with breast cancer were not addressed. Different computational analyses have been introduced to examine SNP-SNP interaction in many association studies [14,16-23]. Genetic algorithm (GA) is potential for feature selection for genome-wide scale datasets [24] and may apply to compute the difference between case and control groups to identify good models from the huge SNP combinations as well as tagSNP selection [25].

To address the possible SNP-SNP interaction in breast cancer susceptibility, five tagSNPs (rs12313273, rs6486795, rs7135617, rs12320939, and rs712853) of ORAI1 gene were selected in this study. Therefore, we introduced the GA to optimizing the analyses of SNP-SNP interactions of ORAI1 gene associated with breast cancer. GA is used to identify the best SNP models (SNP combinations with genotypes) with maximum frequency difference between breast cancer and control groups. Therefore, the best GA-generated SNP models of ORAI1 gene may be useful for predicting the breast cancer risk. 


\section{Methods}

\section{Data set collection}

The case and control subjects are 345 female breast cancer patients and 290 female normal controls where the recruitment was approved by Cancer Center of Kaohsiung Medical University Hospital. The genotype dataset of breast cancer patients of five tagSNPs (rs12313273, rs6486795, rs7135617, rs12320939, and rs712853) of ORAI1 gene with minimum allele frequency (MAF) $>10 \%$ obtained from our previous study [15]. For normal controls, samples of were collected in current study and SNP genotyping was performed as described [15].

\section{Genetic algorithm}

The GA [26] is a well-known evolutionary algorithm, and it has been applied for solving the complex problems in several fields. GA simulates the natural evolution to generate solutions of complex problems, including selection, crossover, mutation, and inheritance. The process of GA has six steps: (1) initializing population, (2) evaluate chromosome values, (3) select two parents using selection operation, (4) crossover operation, (5) mutation operation, and (6) replacement operation.

A population in first step is initialized according encoding schemes of problem. Second step aims to evaluate value of chromosomes in population using fitness function. Third step use the evaluated value of chromosomes to select the two good parents for generating two offspring (step 4). Then firth step is probabilistic to mutate two offspring. Final step is used to improve the value of population. Thus repeat of steps 2 to 6 in several generations can effectively search the good values of chromosomes in population, and a best chromosome in population is regarded to best solution. Algorithm 1 shows the GA pseudo-code, and the below section is detailed to explain the processes of six steps.

Algorithm 1: Genetic algorithm pseudo-code.

01: begin

02: Initializing population according encoding schemes

03: for $g=1$ to the number of generations

04: evaluate chromosomes of population using fitness function

05: select two parents using selection operation

06: generate two offspring using crossover operation

07: mutate two offspring using mutation operation

08: improve the value of population using replacement

09: $\operatorname{next} g$ operation

10: end

\section{Encoding schemes}

A population consists of the several possible solution of problem. The possible solution in GA is named a chromosome that is a set $C=\left\{c_{1}, \ldots, c_{d}\right\}$. In this study, a chromosome indicates a possible model of associations between SNPs. All combinations of SNPs and genotypes can be represented a set $A=S \times G=\{(s, g) \mid s \in S$ and $g \in G\}$ where $S$ is a set of SNPs and $G$ is a set of genotypes. For example, we assume an $S$ contains two SNPs and a $G$ contains three genotypes, i.e., $S=\left\{\mathrm{s}_{1}, \mathrm{~s}_{2}\right\}$ and $G=\left\{\mathrm{g}_{1}, \mathrm{~g}_{2}, \mathrm{~g}_{3}\right\}$. All possible subsets can be represented $A=S \times G=\left\{\left(\mathrm{s}_{1}, \mathrm{~g}_{1}\right)\right.$, $\left.\left(\mathrm{s}_{1}, \mathrm{~g}_{2}\right),\left(\mathrm{s}_{1}, \mathrm{~g}_{3}\right),\left(\mathrm{s}_{2}, \mathrm{~g}_{1}\right),\left(\mathrm{s}_{2}, \mathrm{~g}_{2}\right),\left(\mathrm{s}_{2}, \mathrm{~g}_{3}\right)\right\}$. Each subset in $A$ represents the selected SNP and their genotype. A chromosome is defined $C=\left\{c_{1}, \ldots, c_{d} \mid c_{i}, c_{j} \in A, c_{i} \neq c_{j}, 1 \leq i \neq j \leq d\right\}$ where $d$ is the association model size. A possible chromosome in above example can be assigned as $C=\left\{\left(\mathrm{s}_{1}, \mathrm{~g}_{1}\right)\right.$, $\left.\left(\mathrm{s}_{2}, \mathrm{~g}_{2}\right)\right\}$; it means a model that includes the genotype "AA" of first SNP and the genotype "Aa" of second SNP.

\section{Fitness function}

A value of chromosome $C$ can be evaluated by computing the fitness function; it facilitates GA for eliminating the worst chromosomes of population in each generation. In this study, a total number difference between case data and control data at a model is used to design a fitness function. Equation 1 is used to check a model whether a SNP is repeatedly selected or not. If a SNP is repeatedly selected in a $C$, the value of $C$ is evaluated to zero. If it is not, Equation 2 is used to calculate the total number difference between cases and controls at a model. In Equation 2, the max_P and max_N are a total number of case data and a total number of control data, respectively. The $P$ and $N$ are respectively represented the set of case data and a set of control data; $P_{i}$ is the $i^{\text {th }}$ patient sample in case data and $N_{i}$ is the $i^{\text {th }}$ normal sample in control data. Equation 3 is used to evaluate whether all factors in a model are included in a set of sample. If a sample includes the model, the Equation 3 returns one value into Equation 2; whereas, it returns zero value.

$$
\begin{aligned}
& f(C)= \begin{cases}0 & \text { if } \bigcap_{s_{i} \in C} s_{i} \neq \varphi \\
d(C) & \text { if } \bigcap_{s_{i} \in C} s_{i}=\varphi\end{cases} \\
& d(C)=\frac{\sum_{i=1}^{\max _{-} \mathrm{P}} m\left(C, P_{i}\right)}{\max \_\mathrm{P}}-\frac{\sum_{i=1}^{\max _{-} \mathrm{N}} m\left(C, N_{i}\right)}{\max \_\mathrm{N}} \\
& m(X, Y)= \begin{cases}0 & \text { if } X \nsubseteq Y Y \\
1 & \text { if } X \subseteq Y\end{cases}
\end{aligned}
$$

\section{Selection operation}

Selection operation aims to select the good chromosomes for generating the great offspring; the selected chromosomes name parents. Selection operation in this study uses a rank-based tournament scheme for selecting the two parents. The operation uses fitness function to evaluate all chromosomes of a population $P=\left\{C_{1}, \ldots, C_{i} \mid i\right.$ is population 
size $\}$, and all values in $P$ are recorded into a set $R=\left\{r_{1}, \ldots\right.$, $r_{i} \mid i$ is population size\}. These values represent chromosome ranks. Then $R$ is sorted from the big value to small value, i.e., $r_{1} \geq r_{2} \geq r_{i}$. Thus the $r_{1}$ and $r_{2}$ with corresponding $C$ s in $P$ are two selected parents.

\section{Crossover operation}

Crossover operation is used to generate the offspring from the parents, and the operation use a uniform crossover scheme. Uniform crossover firstly generate a binary mask set $B=\left\{b_{1}, \ldots, b_{i}|b \in[0,1], i=| C \mid\right\}$; a binary value at $b$ is randomly generated. The one value of $b_{j}$ indicates that $j^{\text {th }}$ elements of two parents are must be exchanged; the zero value represents the no exchange. Two offspring are generated by exchanging the elements of two parents according the binary mask set, and the offspring are represented $C$. For example, let a generated binary mask $B=\{1,0,1,0\}$ and two parents $C_{1}=\left\{\left(\mathrm{s}_{1}, \mathrm{~g}_{1}\right),\left(\mathrm{s}_{2}, \mathrm{~g}_{2}\right),\left(\mathrm{s}_{5}, \mathrm{~g}_{1}\right),\left(\mathrm{s}_{3}, \mathrm{~g}_{3}\right)\right\}$ and $C_{2}=\left\{\left(s_{1}, g_{3}\right),\left(s_{2}, g_{1}\right),\left(s_{4}, g_{2}\right),\left(s_{3}, g_{2}\right)\right\}$. The generated two offspring are $C_{1}^{\prime}=\left\{\left(s_{1}, g_{3}\right),\left(s_{2}, g_{2}\right),\left(s_{4}, g_{2}\right),\left(s_{3}, g_{3}\right)\right\}$ and $C_{2}^{\prime}=\left\{\left(s_{1}, g_{1}\right),\left(s_{2}, g_{1}\right),\left(s_{5}, g_{1}\right),\left(s_{3}, g_{2}\right)\right\}$, respectively.

\section{Mutation operation}

Mutation operation can facilitate the diversity of chromosomes in population, and avoid population for trapping the local optimal. The operation uses a one-point mutation operation. A mutation point set $M=\left\{m_{1}, \ldots\right.$, $\left.m_{i}|m \in[0,1], i=| C \mid\right\}$ is used to indicate the mutation points in the offspring $C^{\prime}$. Each binary value in $M$ is randomly generated according to the mutation probability. The one value of $m_{j}$ represents that $j^{\text {th }}$ element of $C$ do the mutation; the zero value represents the no mutation. The mutation randomly generates a possible $c$ element, where $c \in \mathrm{A}$, to replace the original element at a mutation point. For example, let a generated mutation point $M=$ $\{0,0,1,0\}$ and offspring $C_{1}^{\prime}=\left\{\left(s_{1}, g_{3}\right),\left(s_{2}, g_{2}\right),\left(s_{4}, g_{2}\right)\right.$, $\left.\left(s_{3}, g_{3}\right)\right\}$. Suppose the number of SNPs is five, a possible set is $E=\left\{\left(s_{4}, g_{1}\right),\left(s_{4}, g_{2}\right),\left(s_{4}, g_{3}\right),\left(s_{5}, g_{1}\right),\left(s_{5}, g_{2}\right),\left(s_{5}\right.\right.$, $\left.\left.\mathrm{g}_{3}\right)\right\}$. After mutation the offspring could be $C_{1}^{\prime}=\left\{\left(\mathrm{s}_{1}, \mathrm{~g}_{3}\right)\right.$, $\left.\left(s_{2}, g_{2}\right),\left(s_{5}, g_{3}\right),\left(s_{3}, g_{3}\right)\right\}$.

\section{Replacement operation}

Replacement operation aims to gradually improve value of population. The generated two offspring $C_{1}^{\prime}$ and $C_{2}^{\prime}$ are evaluated by fitness function, and are used to compare the value to all chromosomes. When an offspring is higher value than a chromosome of population, it replaces the chromosome; otherwise, the offspring is deleted.

\section{Parameter settings}

In the GA parameters, both of the exchange probabilities in the tournament selection and uniform crossover are 1.0. The exchange probability of a one-point mutation is
0.1. The population size is 50 , and the number of generations is 100 .

\section{Statistical analysis}

All statistical value is computed using SPSS version 19.0 (SPSS Inc., Chicago, IL). Odds ratio (OR) with 95\% confidence interval $(\mathrm{CI})$ is used for measuring a single SNP and the model of association between SNPs; a $P$ value of $<0.05$ is considered statistically significant difference between the cases and controls.

\section{Results}

\section{Data collection}

The complete genotype data set is available at http:// bioinfo.kmu.edu.tw/BRCA-ORAI1-5SNPs.xlsx. Based on these data, the GA-generated SNP models to address the possible SNP-SNP interaction in ORAI1 gene were evaluated in terms of breast cancer association later.

\section{Comparison of patients and normal in terms of effect of single SNP}

Table 1 shows the occurrence of breast cancer for five SNPs in ORAI1 gene. The genotype with major allele ( $G$ in rs12320939; $T$ in rs12313273; $G$ in rs7135617; $T$ in rs6486795; and $\mathrm{T}$ in rs712853) is regarded as the reference for analyzing breast cancer risks in terms of single SNPs. Minor allele is selected according the dbSNP database of NCBI (National Center for Biotechnology Information). No significant differences between the breast cancer patients and controls in all genotypes for each single SNP were found.

Table 1 The performance of five individual SNPs for breast cancer and control groups

\begin{tabular}{lllllll}
\hline SNP ID & Genotype & \multicolumn{2}{l}{ Case (\%) } & \multicolumn{2}{l}{ Control (\%) } & $p$-value \\
\hline 1. rs12320939 & GG & 97 & $(28.12)$ & 79 & $(27.24)$ & \\
& GT & 181 & $(52.46)$ & 140 & $(48.28)$ & 0.785 \\
2. rs12313273 & TT & 183 & $(53.04)$ & 161 & $(55.52)$ & \\
& TC & 142 & $(41.16)$ & 100 & $(34.48)$ & 0.189 \\
& CC & 20 & $(5.80)$ & 29 & $(10.00)$ & 0.107 \\
3. rs7135617 & GG & 103 & $(29.86)$ & 94 & $(32.41)$ & \\
& GT & 187 & $(54.20)$ & 145 & $(50.00)$ & 0.367 \\
& 4T rs6486795 & 55 & $(15.94)$ & 51 & $(17.59)$ & 0.947 \\
& TT & 137 & $(39.71)$ & 121 & $(41.72)$ & \\
& TC & 173 & $(50.14)$ & 126 & $(43.45)$ & 0.260 \\
& CC & 35 & $(10.14)$ & 43 & $(14.83)$ & 0.204 \\
& 5. r712853 & 154 & $(44.64)$ & 128 & $(44.14)$ & \\
& TC & 158 & $(45.80)$ & 134 & $(46.21)$ & 0.904 \\
& CC & 33 & $(9.57)$ & 28 & $(9.66)$ & 0.942 \\
\hline
\end{tabular}


Table 2 The best 10 models in 2-SNP order association

\begin{tabular}{lllll}
\hline $\begin{array}{l}\text { Combined SNP number } \\
\text { (specific SNPs) }\end{array}$ & $\begin{array}{l}\text { SNP } \\
\text { Genotypes }\end{array}$ & $\begin{array}{l}\text { Case } \\
\text { no. }\end{array}$ & $\begin{array}{l}\text { Control } \\
\text { no. }\end{array}$ & $\begin{array}{l}\text { Difference } \\
(\%)^{*}\end{array}$ \\
\hline SNP(1,4) & $2-2$ & 145 & 101 & 7.20 \\
SNP(2,4) & $2-3$ & 22 & 1 & 6.03 \\
SNP(3,4) & $2-2$ & 121 & 85 & 5.76 \\
SNP(2,5) & $2-3$ & 83 & 54 & 5.44 \\
SNP(2,4) & $3-2$ & 65 & 40 & 5.05 \\
SNP(1,5) & $2-3$ & 81 & 55 & 4.51 \\
SNP(4,5) & $2-3$ & 81 & 55 & 4.51 \\
SNP(3,5) & $2-3$ & 78 & 53 & 4.33 \\
SNP(2,3) & $3-3$ & 38 & 23 & 3.08 \\
SNP(4,5) & $2-2$ & 77 & 56 & 3.01 \\
SNP(1,3) & $2-2$ & 147 & 116 & 2.61 \\
\hline
\end{tabular}

*Difference (\%) = Frequency of cases (breast cancer group) - Frequency of controls.

Identification of the best model of SNPs association with maximum frequency difference between breast cancer and control groups

During GA processing, the best ten models of two SNP combinations with genotypes (2-SNP models) were demonstrated in Table 2. In these 2-SNP models, the SNPs $(1,4)$ with genotype 2-2, i.e., [rs12320939-GT]-[rs6486795-TC], possessed the maximum frequency difference (7.20\%) between the breast cancer and control groups, namely the best 2-SNP model. Similarly, the best GA-generated SNP models involving three to five SNP were shown in left side of Table 3.

Odds ratio analysis to identify the best models of SNP associations associated with high breast cancer

Table 3 shows five best models of association involving two to five SNPs. Odds ratio analysis shows the performance (OR, 95\% CI, and $P$ value) of five types of the best models (2- to 5-SNP models) addressing the breast cancer association. The 2-SNP model, i.e., SNPs $(1,4)$ in genotype $2-2$, indicates the borderline significance with breast cancer (OR: 1.357, $P=0.064)$. The 3-SNP model, i.e., SNPs $(1,2,4)$ in genotype 2-1-2, indicates three SNPs (rs12320939, rs12313273, and rs6486795) have a significant association when their genotypes are GT, TT, and TC, respectively (OR: $1.689, P=0.028)$. The 5 -SNP model, SNPs $(1,2,3,4,5)$ in genotype 1-2-3-1-1, indicates all SNPs (rs12320939, rs12313273, rs7135617, rs6486795, and rs712853) have a strongly association when their genotypes are GG, TC, TT, TT, and TT, respectively (OR: 13.148, $P=0.013)$.

\section{Discussion}

GA is a robust non-parametric method that detects nonlinear interactions amongst multiple discrete genetic factors. The advantage of GA is that the method can directly search the good models from the huge number of possible combinations without the training data set. In this study, the fitness function is designed based on the unbalanced data set to compute the difference between case data set and control data set. The function can effectively measure high-risk to search the good model in real data set.

In current study, the $O R$ values of 2- to 3-SNP models are larger than 1 but small, suggesting that the cumulative effect of these four SNPs (rs12320939, rs7135617, rs6486795, and rs712853) are weak. When five SNPs included, the $O R$ value is 13.148 , indicating that the cumulative effect of 5-SNP model becomes strong. This unstable cumulative effect of SNP combinations in SNP models may be partly explained by the experiment design that these five SNPs were only derived from a single gene ORAI1. Because breast cancer is a kind of multigene disease [27-30], therefore, SNPs derived from more genes included in association studies may reveal the cumulative effect effectively [9,11,12,31-33]. Accordingly, the differential performance of the cumulative effects of SNPs from single gene and multigene is worth of further investigation in future.

Table 3 The odds ratio of the best SNP models associated with breast cancer

\begin{tabular}{|c|c|c|c|c|c|c|}
\hline Combined SNP no. (specific SNPs) & SNP Genotypes & Case no./control no. & Cancer (\%) & Difference $(\%)^{*}$ & Odds ratio $(95 \% \mathrm{Cl})$ & $P$ value \\
\hline \multirow[t]{2}{*}{ 2-SNP (1-4) } & $2-2$ & $145 / 101$ & 58.94 & 7.20 & 1.357 & 0.064 \\
\hline & Others & 200/189 & 51.41 & & $(0.983-1.873)$ & \\
\hline \multirow[t]{2}{*}{ 3-SNP (1-2-4) } & $2-1-2$ & $58 / 31$ & 65.17 & 6.12 & 1.689 & 0.028 \\
\hline & Others & $287 / 259$ & 52.56 & & $(1.058-2.694)$ & \\
\hline \multirow[t]{2}{*}{ 4-SNP (1-3-4-5) } & $2-2-2-1$ & $78 / 53$ & 59.54 & 4.33 & 1.306 & 0.180 \\
\hline & others & $267 / 237$ & 52.98 & & $(0.884-1.930)$ & \\
\hline \multirow[t]{2}{*}{ 5-SNP (1-2-3-4-5) } & $1-2-3-1-1$ & $14 / 0$ & 100.00 & 4.06 & 13.148 & 0.013 \\
\hline & Others & $331 / 290$ & 53.30 & & $(1.726-100.142)$ & \\
\hline
\end{tabular}


The computational complexity of GA is calculated by a fitness function of computation. Suppose $n$ iterations is implemented in a test, the computational complexity of GA is $\mathrm{O}(n)$ which represents the big-O in complexity analysis. GA in search of good association model has the below advantages: (1) GA effectively identify the highrisk models in high-order interaction, (2) the best model with statistical significant can be fast identified, and (3) it only has two parameters to need setting and is easily to fulfil for searching the good model. Further, GA is able to analyze high order SNP interactions amongst the huge number of SNPs from GWAS and pharmacogenomics studies in our experiences.

\section{Conclusions}

Although the polymorphisms of ORAI1 gene have been reported to associate with inflammatory diseases [34-36], effects of SNP-SNP interaction to diseases are still unclear. In this study, the GA successfully identified appropriate models of SNP-SNP interactions in breast cancer association study in terms of five SNPs in ORAI1 gene. The resulting SNP models can predict the breast cancer susceptibility more effective than the individual SNPs. This methodology can also apply to any kinds of SNP association studies, such as GWAS, pharmacogenomics and others. Therefore, the possible cumulative effect of SNP combination will be uncovered by this methodology.

\section{Competing interests}

The authors have no competing interests to declare.

\section{Authors' contributions}

W-CC and M-FH carried out the molecular genetic studies. W-CC and Y-YF drafted the manuscript. C-HY instructed $Y$-DL to perform SNP interaction analysis by algorithm. Y-YF, H-WC, and L-YC performed statistics analyses. $\mathrm{H}-\mathrm{WC}, \mathrm{C}-\mathrm{HY}$, and M-FH were involved in discussion and editing the manuscript. All authors read and approved the final manuscript.

\section{Acknowledgements}

This study was partly supported by the National Science Council of Taiwan for Grant NSC101-2221-E-214-075, NSC101-2622-E-151-027-CC3, NSC100-2221-E-151-049-MY3, NSC100-2221-E-151-051-MY2, NSC101-2320B038-029-MY3, the National Sun Yat-sen University-KMU Joint Research Project (\#NSYSU-KMU 103-p014), the Ministry of Health and Welfare, Taiwan, Republic of China (MOHW103-TD-B-111-05), and TMU101-AE1-B14 (to Wei-Chiao Chang).

\footnotetext{
Author details

${ }^{1}$ Master Program for Clinical Pharmacogenomics and Pharmacoproteomics, School of Pharmacy, Taipei Medical University, Taipei, Taiwan. ${ }^{2}$ Department of Clinical Pharmacy, School of Pharmacy, Taipei Medical University, Taipei, Taiwan. ${ }^{3}$ Cancer Center, Kaohsiung Medical University Hospital, Kaohsiung, Taiwan. ${ }^{4}$ Labor Safety and Health Office, Kaohsiung Municipal Ta-Tung Hospital, Kaohsiung, Taiwan. ${ }^{5}$ Department of Biomedical Science and Environmental Biology, Kaohsiung Medical University, Kaohsiung, Taiwan. ${ }^{6}$ Institute of Medical Science and Technology, National Sun Yat-sen University, Kaohsiung, Taiwan. ${ }^{7}$ Department of Chemical Engineering \& Institute of Biotechnology and Chemical Engineering, I-Shou University, Kaohsiung, Taiwan. ${ }^{8}$ Department of Electronic Engineering, National Kaohsiung University of Applied Sciences, Kaohsiung, Taiwan. ${ }^{9}$ Institute of Clinical Medicine, Kaohsiung Medical University, Kaohsiung, Taiwan. ${ }^{10}$ Kaohsiung Municipal Ta-Tung Hospital, Kaohsiung, Taiwan.
}

Received: 2 November 2013 Accepted: 7 March 2014

Published: 31 March 2014

\section{References}

1. Frazer KA, Ballinger DG, Cox DR, Hinds DA, Stuve LL, Gibbs RA, Belmont JW, Boudreau A, Hardenbol P, Leal SM, Pasternak S, Wheeler DA, Willis TD, Yu F, Yang H, Zeng C, Gao Y, Hu H, Hu W, Li C, Lin W, Liu S, Pan H, Tang X, Wang J, Wang W, Yu J, Zhang B, Zhang Q, Zhao H, et al: A second generation human haplotype map of over 3.1 million SNPs. Nature 2007, 449(7164):851-861.

2. Ding $H$, Jing $X$, Ding $N$, Fu Z, Song $Y$, Zhu J: Single nucleotide polymorphisms of CD20 gene and their relationship with clinical efficacy of R-CHOP in patients with diffuse large B cell lymphoma. Cancer Cell Int 2013, 13(1):58.

3. Flintoft L: Complex disease: A SNP for disease prognosis. Nat Rev Genet 2013, 14(11):746.

4. Hwang CW, Lu CH, Sun SF, Sung TY, Chung HY, Huang SY, Hung HC, Chen $\mathrm{CH}$, Sun YM, Lin YY, Liu WS, Wen ZH: Comprehensive association analysis of 10 single nucleotide polymorphisms associated with osteoporosis among a Taiwanese population. Int J Hum Genet 2011, 11(4):249-257.

5. Sober S, Org E, Kepp K, Juhanson P, Eyheramendy S, Gieger C, Lichtner P, Klopp N, Veldre G, Viigimaa M, Doring A, Putku M, Kelgo P, Shaw-Hawkins S, Howard P, Onipinla A, Dobson RJ, Newhouse SJ, Brown M, Dominiczak A, Connell J, Samani N, Farrall M, Caulfield MJ, Munroe PB, Illig T, Wichmann HE, Meitinger T, Laan M: Targeting 160 candidate genes for blood pressure regulation with a genome-wide genotyping array. PLOS One 2009, 4(6):e6034.

6. Kraft $P$, Haiman CA: GWAS identifies a common breast cancer risk allele among BRCA1 carriers. Nat Genet 2010, 42(10):819-820.

7. Lewis SN, Nsoesie E, Weeks C, Qiao D, Zhang L: Prediction of disease and phenotype associations from genome-wide association studies. PLOS One 2011, 6(11):e27175.

8. Yang C, Zhou X, Wan X, Yang Q, Xue H, Yu W: Identifying diseaseassociated SNP clusters via contiguous outlier detection. Bioinformatics 2011, 27(18):2578-2585.

9. Sun J, Chang BL, Isaacs SD, Wiley KE, Wiklund F, Stattin P, Duggan D, Carpten JD, Trock BJ, Partin AW, Walsh PC, Gronberg H, Xu J, Isaacs WB, Zheng SL: Cumulative effect of five genetic variants on prostate cancer risk in multiple study populations. Prostate 2008, 68(12):1257-1262.

10. Yen CY, Liu SY, Chen CH, Tseng HF, Chuang LY, Yang CH, Lin YC, Wen CH, Chiang WF, Ho CH, Chen HC, Wang ST, Lin CW, Chang HW: Combinational polymorphisms of four DNA repair genes XRCC1, XRCC2, XRCC3, and XRCC4 and their association with oral cancer in Taiwan. $J$ Oral Pathol Med 2008, 37(5):271-277.

11. Vogelsang M, Wang Y, Veber N, Mwapagha LM, Parker Ml: The cumulative effects of polymorphisms in the DNA mismatch repair genes and tobacco smoking in oesophageal cancer risk. PLoS One 2012, 7(5):e36962.

12. Yang $\mathrm{CH}$, Chuang LY, Cheng YH, Lin YD, Wang CL, Wen CH, Chang HW: Single nucleotide polymorphism barcoding to evaluate oral cancer risk using odds ratio-based genetic algorithms. Kaohsiung J Med Sci 2012, 28(7):362-368.

13. Kotti S, Bickeboller H, Clerget-Darpoux F: Strategy for detecting susceptibility genes with weak or no marginal effect. Hum Hered 2007, 63(2):85-92.

14. Steen KV: Travelling the world of gene-gene interactions. Brief Bioinform 2012, 13(1):1-19.

15. Chang WC, Woon PY, Hsu YW, Yang S, Chiu YC, Hou MF: The association between single-nucleotide polymorphisms of ORAI1 gene and breast cancer in a Taiwanese population. ScientificWorldJournal 2012, 2012:916587.

16. Yang $\mathrm{CH}$, Chang HW, Cheng YH, Chuang LY: Novel generating protective single nucleotide polymorphism barcode for breast cancer using particle swarm optimization. Canc Epidemiol 2009, 33(2):147-154.

17. Chang HW, Yang CH, Ho CH, Wen CH, Chuang LY: Generating SNP barcode to evaluate SNP-SNP interaction of disease by particle swarm optimization. Comput Biol Chem 2009, 33(1):114-119.

18. Moore JH, Asselbergs FW, Williams SM: Bioinformatics challenges for genome-wide association studies. Bioinformatics 2010, 26(4):445-455

19. Yang CH, Chuang LY, Chen YJ, Tseng HF, Chang HW: Computational analysis of simulated SNP interactions between 26 growth factor-related genes in a breast cancer association study. OMICS 2011, 15(6):399-407. 
20. Chuang LY, Chang HW, Lin MC, Yang CH: Chaotic particle swarm optimization for detecting SNP-SNP interactions for CXCL12-related genes in breast cancer prevention. Eur J Cancer Prev 2012, 21(4):336-342.

21. Yang P, Ho JW, Yang YH, Zhou BB: Gene-gene interaction filtering with ensemble of filters. BMC Bioinforma 2011, 12(Suppl 1):S10.

22. Chuang LY, Lin YD, Chang HW, Yang CH: An improved PSO algorithm for generating protective SNP barcodes in breast cancer. PLOS One 2012, 7(5):e37018.

23. Chuang LY, Chang HW, Lin MC, Yang CH: Improved branch and bound algorithm for detecting SNP-SNP interactions in breast cancer. J Clin Bioinform 2013, 3(1):4.

24. Zhang J, Jiang S, Zhao X, Liu L: A genetic algorithm to filter SNPs for SNP association study. 2012 IEEE/WIC/ACM Int Conferences Web Intelligenl Intell Agent Technol 2012, 1:684-687.

25. Mahdevar G, Zahiri J, Sadeghi M, Nowzari-Dalini A, Ahrabian H: Tag SNP selection via a genetic algorithm. J Biomed Inform 2010, 43(5):800-804.

26. Holland $\mathrm{JH}$ : Adaptation in nature and artificial systems: An introductory analysis with applications to biology, control and artificial intelligence. Ann Arbor, MI: University of Michigan Press; 1975.

27. Solin LJ, Gray R, Baehner FL, Butler SM, Hughes LL, Yoshizawa C, Cherbavaz DB, Shak S, Page DL, Sledge GW Jr, Davidson NE, Ingle JN, Perez EA, Wood WC, Sparano JA, Badve S: A multigene expression assay to predict local recurrence risk for ductal carcinoma in situ of the breast. J Natl Canc Inst 2013, 105(10):701-710.

28. Duggal S, Julian TB: A multigene expression assay to predict local recurrence risk for ductal carcinoma in situ. J Natl Canc Inst 2013, 105(10):681-683.

29. Rouzier R, Pronzato P, Chereau E, Carlson J, Hunt B, Valentine WJ: Multigene assays and molecular markers in breast cancer: systematic review of health economic analyses. Breast Canc Res Treat 2013, 139(3):621-637.

30. Ignatiadis M: Multigene assays for late recurrence of breast cancer. Lancet Oncol 2013, 14(11):1029-1030.

31. Lin GT, Tseng HF, Chang CK, Chuang LY, Liu CS, Yang CH, Tu CJ, Wang EC, Tan HF, Chang CC, Wen CH, Chen HC, Chang HW: SNP combinations in chromosome-wide genes are associated with bone mineral density in Taiwanese women. Chin J Physiol 2008, 51(1):32-41.

32. Lin GT, Tseng HF, Yang CH, Hou MF, Chuang LY, Tai HT, Tai MH, Cheng YH, Wen CH, Liu CS, Huang CJ, Wang CL, Chang HW: Combinational polymorphisms of seven CXCL12-related genes are protective against breast cancer in Taiwan. OMICS 2009, 13(2):165-172.

33. Chen JB, Chuang LY, Lin YD, Liou CW, Lin TK, Lee WC, Cheng BC, Chang $H W$, Yang CH: Preventive SNP-SNP interactions in the mitochondrial displacement loop (D-loop) from chronic dialysis patients. Mitochondrion 2013, 13:698-704.

34. Chang WC, Lee CH, Hirota T, Wang LF, Doi S, Miyatake A, Enomoto T, Tomita K, Sakashita M, Yamada T, Fujieda S, Ebe K, Saeki H, Takeuchi S, Furue M, Chen WC, Chiu YC, Chang WP, Hong CH, Hsi E, Juo SH, Yu HS, Nakamura Y: ORAl1 genetic polymorphisms associated with the susceptibility of atopic dermatitis in Japanese and Taiwanese populations. PLoS One 2012, 7(1):e29387.

35. Chou YH, Juo SH, Chiu YC, Liu ME, Chen WC, Chang CC, Chang WP, Chang JG, Chang WC: A polymorphism of the ORAl1 gene is associated with the risk and recurrence of calcium nephrolithiasis. J Urol 2011, 185(5):1742-1746

36. Wei JC, Yen JH, Juo SH, Chen WC, Wang YS, Chiu YC, Hsieh TJ, Guo YC, Huang $\mathrm{CH}$, Wong RH, Wang HP, Tsai KL, Wu YC, Chang HW, Hsi E, Chang WP, Chang WC: Association of ORAl1 haplotypes with the risk of HLA-B27 positive ankylosing spondylitis. PLoS One 2011, 6(6):e20426.

doi:10.1186/1475-2867-14-29

Cite this article as: Chang et al:: Identifying association model for single-nucleotide polymorphisms of ORAl1 gene for breast cancer. Cancer Cell International 2014 14:29.

\section{Submit your next manuscript to BioMed Central and take full advantage of:}

- Convenient online submission

- Thorough peer review

- No space constraints or color figure charges

- Immediate publication on acceptance

- Inclusion in PubMed, CAS, Scopus and Google Scholar

- Research which is freely available for redistribution

Submit your manuscript at www.biomedcentral.com/submit
C BioMed Central 